\title{
RÉSUMÉS - SOMMAIRE DU GUIDE POUR LE DÉPISTAGE ET LE DIAGNOSTIC DE L'INFECTION PAR LE VIRUS DE L'IMMUNODÉFICIENCE HUMAINE (VIH)
}

\author{
GALE-ROWE M., DODDS J., PAQUETTE D. ET WONG T. AU NOM DU GROUPE DE TRAVAIL \\ D'EXPERTS DE L'ASPC*
}

MOTS-CLÉS : VIH/SIDA, DÉPISTAGE, TEST DE DÉPISTAGE DU VIH

\section{Résumé structuré}

\section{CONTEXTE}

L'Agence de la santé publique du Canada (ASPC) estime qu'en 2011, $25 \%$ des personnes vivant avec le VIH au Canada n'avaient pas été diagnostiquées. L'hésitation à faire un test de dépistage peut provenir de la crainte, de la stigmatisation et de la discrimination associées à un diagnostic d'une infection par le VIH et aux comportements à risque connexes. Le présent guide a pour objectif de contribuer aux efforts existants visant à soutenir les fournisseurs de soins directement concernés par le dépistage du $\mathrm{VIH}$, dans le but de réduire le nombre d'infections par le VIH non diagnostiquées au Canada.

\section{DÉMARCHE}

L'ASPC a sollicité une analyse documentaire et a consulté les responsables des provinces et des territoires, ainsi que des acteurs clés, notamment des personnes vivant avec le $\mathrm{VIH} /$ sida, des intervenants du milieu universitaire, des infirmiers, des médecins, des associations professionnelles, des organisations non gouvernementales, des responsables des politiques, des travailleurs communautaires et des spécialistes des questions juridiques et éthiques. Les recommandations formulées dans le guide reposent donc sur les données les plus récentes et l'opinion d'experts.

\section{GUIDE POUR LE DÉPISTAGE ET LE DIAGNOSTIC}

Le dépistage du VIH devrait être offert et faire l'objet de discussion dans le cadre des soins de santé réguliers. En offrant couramment le test de dépistage du $\mathrm{VIH}$, il est possible de normaliser le dépistage et de faire disparaître les multiples obstacles à la réduction du nombre de cas non diagnostiqués au Canada. Une brève explication du mode de transmission du VIH est d'abord donnée au client : au cours de relations sexuelles non protégées, par le partage de matériel de consommation de drogues, et d'une mère à son bébé au cours de la grossesse. Les clients peuvent alors évaluer leur propre situation et indiquer s'ils désirent subir un test de dépistage du VIH. Une évaluation du risque peut être réalisée sur demande. Tout comme pour les autres tests, le dépistage est volontaire et l'obtention du consentement verbal est suffisante.Les résultats négatifs offrent une occasion de rappeler aux clients les pratiques qui peuvent les aider à prévenir l'infection par le VIH. II existe toute une gamme de références et de ressources disponibles pour aider les clients à réduire les activités à risque et à rester séronégatifs. Les clients qui sont en couple doivent être encouragés à discuter du dépistage du VIH avec leur partenaire afin d'éviter de vivre une relation sérodiscordante à leur insu.

Les résultats positifs du test devraient toujours être communiqués en personne, idéalement par le fournisseur de soins initialement consulté qui aura à portée de main la documentation et les services d'orientation pertinents. 
Il peut être difficile de recevoir un diagnostic de séropositivité; il est important de prévoir suffisamment de temps pour discuter des résultats et répondre aux questions que le client pourrait poser. L'accent doit être mis sur les messages positifs en soulignant les progrès réalisés dans la prise en charge, le traitement et le soutien des personnes infectées par le $\mathrm{VIH}$. II faut mentionner que le $\mathrm{VIH}$ est maintenant considéré comme une maladie chronique, et que les personnes vivant avec le VIH peuvent jouir d'une vie longue, active et saine. Le client doit être avisé des stratégies de prise en charge du VIH et orienté vers les soins requis. De l'information sur la réduction des risques pour prévenir la transmission du virus doit aussi lui être fournie. Les clients dont les résultats sont positifs doivent savoir que ceux-ci seront partagés confidentiellement avec le service de santé publique local, qui peut aider à aviser les partenaires actuels ou précédents qu'ils doivent procéder au dépistage, tout en protégeant l'anonymat et la vie privée du client. Les stratégies pour informer les partenaires antérieurs, actuels et éventuels peuvent être examinées. Si cela n'a pas déjà été réalisé dans le cadre du dépistage du VIH, le client doit procéder au dépistage d'autres infections transmissibles sexuellement (ITS), des hépatites $B$ et $C$ et de la tuberculose.

\section{Introduction}

II est essentiel d'optimiser les occasions pour les fournisseurs de soins de proposer le dépistage du VIH aux personnes vivant au Canada.

Les cas non diagnostiqués sont des occasions manquées de réduire la transmission du VIH et d'améliorer la productivité, le pronostic de la maladie et la qualité de vie des personnes vivant avec le virus. L'Agence de la santé publique du Canada estime qu'en 2011, 25 \% des personnes vivant avec le VIH au Canada ignoraient qu'elles étaient infectées (1).

La réduction du nombre d'infections au VIH non diagnostiquées aurait plusieurs avantages. Un résultat négatif du test offre au client l'occasion de prendre des mesures pour préserver sa séronégativité. La morbidité et la mortalité associées à l'infection au VIH ainsi que la progression de la maladie peuvent être réduites chez les personnes qui obtiennent un diagnostic précoce et qui amorcent le traitement antirétroviral hautement actif (HAART), comparativement à celles dont le diagnostic n'est pas établi en temps opportun (2). Les progrès réalisés dans le traitement du VIH ont ralenti l'évolution de la maladie au point que l'infection au VIH est désormais considérée comme une affection chronique et maîtrisable, qui permet à un plus grand nombre de personnes séropositives de vivre une vie active et saine pendant de longues années.

Les personnes dont le résultat du test est positif sont plus susceptibles de prendre des mesures permettant de prévenir la propagation du $\mathrm{VIH}$ (3). Des données récentes montrent qu'une charge virale réduite grâce au HAART amorcé rapidement, combinée à d'autres moyens de prévention tels que l'utilisation systématique du condom, est associée à une baisse relative de $96 \%$ des transmissions sexuelles du $\mathrm{VIH}-1$ dans certaines populations par rapport au traitement retardé $(4,5)$.

En revanche, les personnes qui ne sont pas au courant de leur infection au VIH sont plus susceptibles de présenter une morbidité et une mortalité accrues et de propager le virus à leur insu.

Malgré les avantages liés au dépistage du VIH, il est possible que la crainte d'avoir un résultat positif et la crainte de subir de la discrimination à la suite d'un diagnostic d'une infection par le VIH soient des obstacles au dépistage (6). Pour réduire le nombre d'infections par le VIH non diagnostiquées, il faut en arriver à un équilibre entre les tests ciblant les personnes les plus à risque et une stratégie moins ciblée visant les populations à " risque modéré ». Les données montrent que de nombreuses personnes qui ne font pas partie des populations à haut risque classiques, ou ne sont pas perçues comme telles, ne se font pas offrir le dépistage du VIH $(7,8)$. Pour cette raison, il arrive parfois qu'elles obtiennent un diagnostic au moment où la maladie a beaucoup progressé, même si elles ont eu de nombreuses interactions avec des professionnels de la santé (8).

Le présent document est un sommaire du Guide pour le dépistage et le diagnostic de l'infection par le VIH (9). 


\section{Démarche}

Afin d'éclairer la rédaction du présent guide, l'Agence a sollicité une analyse documentaire et a fait des consultations auprès des responsables des provinces et des territoires, ainsi que des acteurs clés, notamment des personnes vivant avec le $\mathrm{VIH} /$ sida et d'autres populations concernées, des intervenants du milieu universitaire, des infirmiers, des médecins, des associations professionnelles, des organisations non gouvernementales, des responsables des politiques, des travailleurs communautaires et des spécialistes des questions juridiques et éthiques. Les recommandations formulées dans le guide reposent donc sur les données les plus récentes et l'opinion d'experts.

II faut noter que ce guide ne remplace pas les exigences provinciales ou territoriales en matière de réglementation, de politiques et de pratique, ni les lignes directrices professionnelles qui régissent et guident la pratique des fournisseurs de soins de santé dans leur province ou territoire respectif. Comme toujours, les fournisseurs de soins doivent respecter les exigences en matière de pratique et les règlements locaux en matière de santé publique lorsqu'ils réalisent un dépistage du $\mathrm{VIH}$. Le présent guide vise à offrir des conseils supplémentaires dans le but de réduire le nombre d'infections par le VIH non diagnostiquées au Canada.

\section{Recommandations}

Le présent guide est axé sur de nombreux principes directeurs. Le premier principe est l'importance que revêtent la promotion et la protection de la santé publique, ce qui comprend la nécessité d'intégrer le dépistage du VIH aux services de dépistage d'autres infections connexes comme les infections transmissibles sexuellement et par le sang et la tuberculose. Le deuxième principe est l'importance des droits de la personne, ce qui comprend les « $3 \mathrm{C}$ » du dépistage du VIH : counseling, consentement éclairé et confidentialité. Le troisième est le principe de l'adaptabilité, qui reflète la nécessité de mettre en œuvre les recommandations avec souplesse en fonction des besoins des clients et des fournisseurs de soins

\section{NORMALISATION DU DÉPISTAGE DU VIH}

Il est recommandé d'offrir le dépistage du VIH et d'en discuter dans le cadre des soins de santé réguliers. Cette recommandation est basée sur deux observations. Premièrement, les données laissent croire que le risque perçu ne correspond pas toujours avec exactitude au risque réel et, lorsqu'il est combiné à la crainte, peut réduire la probabilité que le patient demande lui-même un dépistage du VIH (6). Deuxièmement, des données de qualité démontrent les bienfaits associés à la normalisation du dépistage du VIH comme moyen pour surpasser les multiples obstacles au dépistage du VIH (10) (Tableau 1). Le dépistage permet à tous les clients d'atténuer l'angoisse liée à la séro-ignorance et d'établir des données de base dans le cadre du programme de soins de santé de chacun. II faudrait offrir aux personnes ayant des comportements à risque élevé de subir un dépistage du VIH au moins une fois par an.

Une évaluation approfondie et complète des risques de VIH liés au comportement n'est pas une condition préalable pour offrir un test de dépistage du VIH. Une évaluation de la capacité du client à comprendre la façon dont le VIH est transmis et les conséquences du dépistage (avantages et inconvénients) et à interpréter la signification des résultats du test est suffisante. Le dépistage à l'initiative du fournisseur de soins peut commencer par une brève explication fournie au client sur le mode de transmission du VIH : au cours de relations sexuelles non protégées, lors du partage de matériel de consommation de drogues et d'une mère à son bébé pendant la grossesse. Les clients peuvent alors évaluer leur propre situation et indiquer s'ils désirent subir un test de dépistage du VIH.

Il est utile de disposer de ressources imprimées ou en ligne pour soutenir les besoins d'information des clients et pour éclairer les discussions et les décisions relatives au dépistage du VIH. Dans les cas où les clients ne sont pas en mesure d'évaluer avec précision leur risque, un examen des indications cliniques du test de dépistage du VIH et des facteurs de risque du virus peut être utile. (Tableau 2) Rarement, une évaluation approfondie des risques liés au comportement peut être nécessaire (9). II faut aviser le client de son droit de refuser le test. Le dépistage du VIH reste volontaire et fondé sur un consentement éclairé. Un consentement écrit n'est pas nécessaire. 
Afin de surmonter les problèmes de stigmatisation, il importe que les fournisseurs de soins respectent les diversités culturelles, sexuelles et liées au genre des clients en adoptant un langage, des comportements et des attitudes sans porter de jugement, et qu'ils utilisent les ressources d'information appropriées pour illustrer ces diversités.

II pourrait être avantageux pour les personnes qui présentent un risque d'exposition au VIH et qui ont des relations sexuelles avec un partenaire régulier de se voir offrir le dépistage du VIH en couple. Les données révèlent que les couples qui subissent ensemble le dépistage et qui connaissent mutuellement leurs résultats sont plus susceptibles d'adopter des comportements qui protégeront leur partenaire, comparativement à ceux qui effectuent le dépistage seuls (11). Le dépistage en couple réduit la transmission du VIH au sein des couples sérodifférents qui ignorent leur état sérologique et le risque de transmission ou d'acquisition du VIH avec des partenaires sexuels extérieurs au couple.

\section{COUNSELING PRÉ-TEST}

II est utile de fournir aux clients de l'information sur le VIH dans la salle d'attente pour les aider à se préparer à l'entretien avant le test. Les clients qui obtiennent un résultat positif au dépistage étant moins susceptibles de retenir l'information clé du counseling post-test, certains des renseignements fournis après le test sont intégrés à la discussion préalable au test.

Communiquer un message positif sur les avantages de savoir que l'on est séronégatif ou sur les avantages d'un diagnostic précoce, notamment les traitements disponibles et un meilleur pronostic de la maladie. Expliquer en quoi consiste la fenêtre sérologique et, si des comportements à risque ont été adoptés au cours des trois derniers mois, indiquer qu'un test de suivi pourrait être nécessaire. Discuter des mesures que le client peut prendre pour éviter de contracter ou de transmettre l'infection par le VIH ou d'autres infections transmissibles sexuellement et par le sang, et expliquer que ces mesures devraient être appliquées jusqu'à ce que tous les tests aient été effectués (lorsqu'un comportement à risque est soutenu, le client reste continuellement dans la fenêtre sérologique). Assurer au client devant subir le test que son droit à la vie privée ou son anonymat seront préservés. Lui expliquer les limites de la confidentialité, notamment que, si le résultat est positif, il sera déclaré de manière confidentielle au service de santé publique local. Aviser le client des avantages pour la santé publique à divulguer sa séropositivité à ses partenaires actuels et futurs si le résultat du test est positif. Définir les besoins de soutien du client après la réalisation du dépistage. En général, les résultats du test de dépistage du VIH devraient être communiqués en personne, mais il peut être bon d'avoir un autre mode de communication convenu pour annoncer un résultat négatif et présenter des recommandations de suivi, comme un appel téléphonique sécurisé, une lettre ou un courriel.

\section{TEST DE DÉPISTAGE}

Des algorithmes de dépistage du VIH ont été établis afin d'optimiser les valeurs prédictives positives et négatives. Une discussion détaillée des tests va au-delà du présent guide. Pour obtenir des renseignements propres à son secteur, il faut communiquer avec le laboratoire de santé publique local. Toutefois, il importe de souligner que le counseling pré- et post-test doit être adapté à la génération et au type de test. Par exemple, la fenêtre sérologique des dernières générations de tests est moindre par rapport à celle des générations précédentes. Dans le cas du dépistage rapide, comparativement au test effectué en laboratoire, une évaluation est réalisée pour savoir si le client est prêt à recevoir un résultat de test au cours de la même séance et s'il comprend la signification des résultats non réactifs et réactifs ainsi que la possibilité d'un faux positif. Santé Canada exige que les trousses de dépistage rapide soient uniquement utilisées dans les contextes où un counseling préalable et postérieur au test de dépistage du VIH est offert.

\section{COUNSELING POST-TEST}

Les messages clés à l'intention des clients doivent être adaptés au résultat du test de dépistage du VIH et à chaque situation. Parfois, les activités avant et après le test peuvent être entreprises durant une seule séance. Le niveau de soutien requis dans un contexte de dépistage donné peut englober de l'information, des discussions, du counseling et un renvoi. II faudrait offrir à tous les clients des services d'aiguillage et de soutien, quels que soient leurs résultats de test, pour appuyer les mesures de réduction du risque. II est possible de trouver ces services en communiquant 
avec les services de santé publique locaux ou les centres de crise locaux, ou en composant le numéro des lignes info-santé provinciales ou territoriales ou des services d'écoute sida.

\section{RÉSULTAT INDÉTERMINÉ}

Lorsqu'un résultat est indéterminé, il faut réaliser d'autres tests. Conseiller au client de conserver les pratiques de réduction des risques jusqu'à ce que le dépistage soit terminé. Passer en revue les messages communiqués pendant le counseling pré-test.

\section{RÉSULTAT NÉGATIF}

Dans la plupart des cas, le résultat du test de dépistage du VIH sera négatif. Les clients doivent être informés de la fenêtre sérologique et, si des comportements à risque ont été adoptés au cours des trois mois précédant le test, un test de suivi est recommandé. La confirmation de résultats négatifs offre une occasion de rappeler aux clients les pratiques qui peuvent les aider à rester séronégatifs. Les clients qui reçoivent un résultat négatif et qui sont en couple doivent être encouragés à discuter du dépistage du VIH avec leur partenaire et les personnes avec qui ils consomment des drogues.

\section{RÉSULTAT POSITIF}

Un résultat positif au dépistage du VIH doit toujours être communiqué en personne et idéalement par le fournisseur de soins initialement consulté. Les fournisseurs de soins doivent se préparer en mettant la documentation et les services d'orientation pertinents à la disposition du client et doivent prévoir suffisamment de temps pour discuter des résultats et répondre aux questions immédiates que le client pourrait poser. Les clients entendent souvent seulement le résultat positif; il peut donc être utile de prévoir un rendez-vous de suivi dans les deux semaines afin de poursuivre la discussion.

II convient de rassurer les clients sur le fait que leur vie privée et la confidentialité des renseignements à leur sujet seront protégées. II faut mentionner que grâce à un traitement et à des soins adéquats, les personnes atteintes du $\mathrm{VIH}$ peuvent jouir d'une vie longue, active et saine, et les clients doivent savoir que le VIH est maintenant considéré comme une maladie chronique maîtrisable. Le client doit être avisé des stratégies de prise en charge du VIH et orienté vers les soins requis. Si cela n'a pas déjà été réalisé dans le cadre du dépistage du $\mathrm{VIH}$, le client doit procéder au dépistage d'autres infections transmissibles sexuellement (ITS), des hépatites $B$ et $C$ et de la tuberculose. Les tests de base, telles la numération des lymphocytes CD4, la mesure de la charge virale plasmatique et les épreuves de sensibilité aux médicaments, doivent être réalisés. II est impératif que les clients soient informés de la façon de prévenir une plus ample propagation du virus.

Un plan de notification aux partenaires doit être élaboré avec le client de façon à aviser les partenaires précédents et actuels qu'ils doivent effectuer un dépistage. Plusieurs options existent pour la notification aux partenaires. Avec le consentement du client, les services de santé publique peuvent aider à informer dans l'anonymat les partenaires sexuels et les partenaires de consommation de drogues potentiellement infectés ayant été identifiés par le client. Par ailleurs, le fournisseur qui réalise le test peut aider le client à informer ses partenaires anonymement ou il se peut que le client veuille communiquer directement avec ses partenaires. Certains clients préfèrent une combinaison de ces options, selon la personne à contacter. Dans les cas où les partenaires sexuels se sont rencontrés par le truchement d'Internet ou ont seulement échangé une adresse de courriel ou un nom d'utilisateur, des outils Web tels que inSPOT peuvent accroître la notification des partenaires. Un délai devrait être convenu pour confirmer que les partenaires ont été avisés.

Le fait de fournir de l'information sur la réduction des risques pour prévenir la transmission du virus est un élément essentiel des procédures à suivre après le test. Dans les cas où le fournisseur de soins n'est pas en mesure de fournir de l'information détaillée sur la réduction des risques, il doit orienter le client vers des services de réduction des risques. 
Proposer au client de l'aiguiller vers des services de counseling spécialisés équipés pour fournir aux personnes ayant récemment reçu un diagnostic de séropositivité, le soutien et les ressources précis dont elles ont besoin pour prendre en charge leur santé et leur bien-être. Des services d'écoute $\mathrm{VIH} /$ sida sont offerts dans chaque province et territoire au Canada.

\section{Conclusion}

Le présent guide a été conçu pour aider les fournisseurs de soins à offrir le dépistage du VIH couramment et avec souplesse en vue de dépister des cas de $\mathrm{VIH}$ non diagnostiqués auparavant. II sera ainsi possible de réduire la transmission du VIH et d'améliorer la productivité, le pronostic de la maladie et la qualité de vie des personnes aux prises avec le VIH. Une fois le diagnostic établi, des services de soutien et des soins continus sont essentiels.

\section{Références}

1. Agence de la santé publique du Canada. Résumé : Estimations de la prévalence et de l'incidence du VIH au Canada, 2011. Ottawa (Ont.).

2. Jain V, Deeks SG. When to start antiretroviral therapy. Current HIVIAIDS Reports. 2010;7(2):60-8.

3. Marks G, Crepaz N, Senterfitt JW, Janssen RS. Meta-analysis of high-risk sexual behavior in persons aware and unaware they are infected with HIV in the united states: Implications for HIV prevention programs. J Acquir Immune Defic Syndr. 2005 Aug 1;39(4):446-53.

4. Attia S, Egger M, Müller M, Zwahlen M, Low N. Sexual transmission of HIV according to viral load and antiretroviral therapy: Systematic review and meta-analysis. AIDS. 2009;23(11):1397-404.

5. Cohen MS, Chen YQ, McCauley M, Gamble T, Hosseinipour MC, Kumarasamy N, et al. Prevention of HIV-1 infection with early antiretroviral therapy. N Engl J Med. 2011;365(6):493-505.

6. Deblonde J, De Koker P, Hamers FF, Fontaine J, Luchters S, Temmerman M. Barriers to HIV testing in europe: A systematic review. Eur J Public Health. 2010;20(4):422-32.

7. Burke RC, Sepkowitz KA, Bernstein KT, Karpati AM, Myers JE, Tsoi BW, et al. Why don't physicians test for HIV? A review of the US literature. AIDS. 2007;21(12):1617-24.

8. Girardi E, Sabin CA, Monforte AD. Late diagnosis of HIV infection: Epidemiological features, consequences and strategies to encourage earlier testing. J Acquir Immune Defic Syndr. 2007;46(SUPPL. 1):S3-8.

9. Agence de la santé publique du Canada. Virus de l'immunodéficience humaine - Guide pour le dépistage et le diagnostic de l'infection par le VIH. Canada : 2013. http://publications.gc.ca/collections/collection_2013/aspc-phac/HP40-76-2012fra.pdf

10. European Centre for Disease Prevention and Control. HIV testing: Increasing uptake and effectiveness in the European Union. Stockholm: ECDC; 2010.

11. World Health Organization. Guidance on couples HIV testing and counselling including antiretroviral therapy for treatment and prevention in serodiscordant couples: Recommendations for a public health approach. Geneva: WHO; 2012.

\section{Remerciements}

L'Agence de la santé publique du Canada désire remercier les membres du Comité directeur sur les maladies transmissibles et infectieuses, le Comité consultatif fédéral-provincial-territorial sur le sida, le Groupe de travail d'experts - Lignes directrices canadiennes sur les infections transmissibles sexuellement, le Conseil des médecins hygiénistes en chef, le Réseau des laboratoires de santé publique du Canada et la Canadian Association of HIV Clinical Laboratory Specialists pour leur contribution à ce présent guide. L'ASPC désire également remercier tout le personnel du Centre de la lutte contre les maladies transmissibles et les infections et du Laboratoire national de microbiologie qui ont participé à l'élaboration de ce document. 


\section{Déclaration de conflit d'intérêts}

II n'y a aucun conflit d'intérêts à déclarer.

\section{Financement}

Ce travail a été appuyé par l'Agence de la santé publique du Canada.

\section{TABLEAU 1. OBSTACLES AU DÉPISTAGE DU VIH ET RECOMMANDATIONS PRÉSENTÉES DANS LE GUIDE POUR LES SURMONTER}

\begin{abstract}
Incapacité d'évaluer avec exactitude le risque d'exposition au VIH par certains clients et fournisseurs de soins

Normaliser le dépistage du VIH; simplifier les évaluations du risque; offrir le dépistage du VIH dans le cadre des soins de santé réguliers
\end{abstract}

Gêne à parler du dépistage du VIH et de l'infection elle-même chez certains clients et fournisseurs de soins

Manque de temps de la part du fournisseur de soins pour les évaluations du risque et le counseling pré- et post-test

Procédure fastidieuse pour obtenir le consentement

Crainte de la stigmatisation et de la discrimination associées aux comportements à risque et aux résultats positifs
Normaliser le dépistage du VIH; simplifier les évaluations du risque; offrir le dépistage du VIH dans le cadre des soins de santé réguliers

Simplifier les évaluations du risque; utiliser divers moyens pour transmettre l'information pré-test (documents écrits, vidéos, ressources mobiles, documents Web); offrir des moyens différents pour transmettre les résultats négatifs

Le consentement verbal au dépistage du VIH, tout comme aux autres tests, est suffisant; le dépistage demeure volontaire

Normaliser le dépistage du VIH et simplifier l'évaluation du risque afin de réduire la gêne et la stigmatisation et d'augmenter l'acceptation du test; souligner le fait que l'infection par le VIH est une maladie chronique maîtrisable et décrire les avantages du traitement afin de réduire la crainte d'obtenir un résultat positif

\section{TABLEAU 2. INDICATIONS CLINIQUES DU TEST DE DÉPISTAGE DU VIH ET FACTEURS DE RISQUE} D'INFECTION PAR LE VIH

\section{INDICATIONS CLINIQUES DU TEST DE DÉPISTAGE DU VIH}

1. Personnes qui demandent un dépistage du VIH.

2. Personnes présentant les signes et symptômes de l'infection au $\mathrm{VIH}$.

3. Personnes souffrant de maladies associées à un système immunitaire affaibli ou à un diagnostic de tuberculose.

4. Relations sexuelles anales ou vaginales non protégées ou partage de matériel de consommation de drogues avec un partenaire sachant qu'il est séropositif.

5. Femmes enceintes ou envisageant de l'être; et leurs partenaires, le cas échéant.

6. Victimes d'agression sexuelle

\section{FACTEURS AUGMENTANT LE RISQUE D'INFECTION AU VIH}

1. Personne sexuellement active, mais sans antécédents de dépistage du VIH.

2. Partage de matériel de consommation de drogues avec un partenaire dont l'état sérologique n'est pas connu.

3. Relations sexuelles anales ou vaginales avec un partenaire dont l'état sérologique n'est pas connu.

4. Partenaires sexuels multiples ou anonymes.

5. Pour les hommes, antécédents de relations sexuelles avec un autre homme.

6. Diagnostic d'autres infections transmissibles sexuellement, d'hépatite B ou C.

7. Activité sexuelle, partage de matériel de consommation de drogues, ou réception d'une transfusion sanguine ou de produits sanguins pour les personnes venant de régions où le $\mathrm{VIH}$ est endémique ou y ayant voyagé. 
TABLEAU 2. INDICATIONS CLINIQUES DU TEST DE DÉPISTAGE DU VIH ET FACTEURS DE RISQUE D'INFECTION PAR LE VIH

INDICATIONS CLINIQUES DU TEST DE DÉPISTAGE DU VIH

8. Réception d'une transfusion sanguine ou de produits sanguins au Canada avant novembre 1985. 